\title{
REVERSIBILITY AND ENTROPY PRODUCTION OF INHOMOGENEOUS MARKOV CHAINS
}

\author{
$\mathrm{HAO} \mathrm{GE},{ }^{* * *}$ \\ DA-QUAN JIANG **** AND \\ MIN QIAN, ${ }^{*}$ Peking University
}

\begin{abstract}
In this paper we introduce the concepts of instantaneous reversibility and instantaneous entropy production rate for inhomogeneous Markov chains with denumerable state spaces. The following statements are proved to be equivalent: the inhomogeneous Markov chain is instantaneously reversible; it is in detailed balance; its entropy production rate vanishes. In particular, for a time-periodic birth-death chain, which can be regarded as a simple version of a physical model (Brownian motors), we prove that its rotation number is 0 when it is instantaneously reversible or periodically reversible. Hence, in our model of Markov chains, the directed transport phenomenon of Brownian motors can occur only in nonequilibrium and irreversible systems.
\end{abstract}

Keywords: Brownian motor; inhomogeneous Markov chain; instantaneous reversibility; instantaneous entropy production; rotation number

2000 Mathematics Subject Classification: Primary 60J27

Secondary $82 \mathrm{C} 31 ; 82 \mathrm{C} 70$

\section{Introduction}

The concept of entropy production was first put forward in nonequilibrium statistical physics to describe how far a specific state of a system is from its equilibrium state [10]-[13], [20], [26]. It is closely related to macroscopic irreversibility, another concept in nonequilibrium statistical physics. A macroscopic irreversible system in a steady state should have positive entropy production rate and should be in nonequilibrium.

In earlier work, [21]-[24], various authors defined the entropy production rate of a stochastic process measure theoretically as the specific relative entropy of the distribution of the process with respect to that of its time reverse. Moreover, the formulae for entropy production rate and circulation distribution of homogeneous Markov chains, Q-processes, and diffusions were derived and their relationship with reversibility discussed: the chain or process is reversible if and only if its entropy production vanishes, or if and only if there is no net circulation. All of this work is now included in [15].

The notion of instantaneous entropy production was first put forward in [22], which is also to be found in [15, p. 102]. Recently, Zhang [31] used the instantaneous entropy production rate at time 0 of Markov chains to calculate the entropy production of exclusion processes on groups. Until now, however, the mathematics of instantaneous reversibility and instantaneous entropy production has not been rigorously studied.

Received 13 May 2006; revision received 12 September 2006.

* Postal address: LMAM, School of Mathematical Sciences, Peking University, Beijing, 100871, P. R. China.

** Email address: gehao@math.pku.edu.cn

*** Email address: jiangdq@ math.pku.edu.cn 
In the last three decades, the phenomenon of Brownian motors (sometimes called ratchet systems) has attracted much interest from physicists and biochemists [2]-[5], [16], [18], [19]. A net current of particles can be driven by noise, providing that there is an appropriate asymmetry in the system, such as a spatially periodic and asymmetric, so-called ratchet, potential. Reimann [25] reviewed in detail the theoretical models and experimental realizations of this phenomenon of noise-driven mass transport in spatially periodic systems out of thermal equilibrium. In many situations, the ratchet system may be subjected to time-periodic drive (e.g. rocking ratchets), the temperature of the thermal noise may be subjected to periodic temporal variations (e.g. temperature ratchets), or the potential may fluctuate periodically (e.g. on-off ratchets). As a result, we consider a stochastic differential equation of the form

$$
\mathrm{d} X_{t}=b\left(t, X_{t}\right) \mathrm{d} t+\sigma\left(t, X_{t}\right) \mathrm{d} W_{t},
$$

in which $W_{t}$ is a Brownian motion and $b(t, x)$ and $\sigma(t, x)$ are periodic in the time parameter $t$.

Such stochastic differential equations also appear in the phenomenon of stochastic resonance, in which a weak periodic signal in a nonlinear system can be amplified by added noise. An extensive description of this phenomenon from the physical point of view can be found in [8]. Noise plays a creative role in the phenomena of both Brownian motors and stochastic resonance. However, the time inhomogeneity of (1.1) causes many difficulties in studying the properties of its solution process mathematically.

In this article we will consider a discrete variant of (1.1), namely a continuous-time Markov chain with time-periodic transition densities, which we will call a periodically inhomogeneous Markov chain. Such a Markov chain arises in the numerical method for solving (1.1) which was introduced in [7] and developed in [1], [28], and [29]. This spatially discretized model also arises in the context of the activated barrier-crossing limit, as discussed in [25, Sections 3.8 and 6.7].

In [9] the authors discussed another, simpler, discrete model of Brownian motors, namely discrete-time Markov chains with periodically varying transition matrices, introducing the concepts of periodic reversibility, periodic entropy production rate, and circulation distribution. The main result of [9] is the equivalence of the following statements: the time-periodic Markov chain is periodically reversible; it is in detailed balance; Kolmogorov's cycle condition is satisfied; its entropy production rate vanishes; every circuit and its reversed circuit have the same circulation weight.

In this paper we extend the notions and results of [15] and [21]-[24] to the situation of a general inhomogeneous Markov chain whose definition and construction are given in Section 2. In Section 3 we introduce for it the notion of instantaneous reversibility, define its instantaneous entropy production rate from the physical point of view, and discuss the relationship between these two notions. From this, we obtain the main result of this article, as follows. By $\pi(s)=$ $\left(\pi_{i}(s)\right)_{i \in S}$ we denote the distribution of $X(s)$, and by $Q(s)=\left(q_{i j}(s)\right)_{i, j \in S}$ we denote its transition density matrix function at time $s$.

Theorem 1.1. The following statements are equivalent, in close accord with physical theory.

1. An inhomogeneous Markov chain X is instantaneously reversible at time s.

2. It is in detailed balance at time $s: \pi_{i}(s) q_{i j}(s)=\pi_{j}(s) q_{j i}(s)$ for all $i, j \in S$.

3. Its instantaneous entropy production rate at time $s$ vanishes. 
In the latter part of Section 3 we consider periodically inhomogeneous Markov chains including birth-death chains on a ring, which are the discrete variant of Brownian motors (see (1.1)). We introduce another concept of reversibility, so-called 'periodic reversibility', first put forward in [9]. We prove that when a time-periodic birth-death chain is either instantaneously reversible or periodically reversible for some index, its rotation number is 0 . The rotation number of a birth-death chain or a diffusion process corresponds to the average particle current, which is the quantity of central interest in Brownian motors [15, Chapters 1, 2, and 5], [25]. Hence, in our Markov chain model, the directed transport phenomenon of Brownian motors can occur only in nonequilibrium and irreversible systems.

In Section 4 we give the measure-theoretical definition of the instantaneous entropy production rate of an inhomogeneous Markov chain. The main idea and method come from [15, Chapter 2]. Section 5 contains some remarks and examples, and also some analogous results for inhomogeneous, discrete-time Markov chains. We believe that the results can be extended to inhomogeneous diffusion processes, though much technical work remains to be done.

\section{Definition and construction}

The main results in this section come from [14], [17], and [30, pp. 16-21]

Definition 2.1. (Transition density matrices ( $Q$-property).) If the matrices

$$
\left\{Q(t)=\left(q_{i j}(t)\right)_{i, j \in S}: t \geq 0\right\}
$$

with $S=\{1,2, \ldots, N\}, N \leq \infty$, are such that

1. $0 \leq q_{i j}(t)<\infty$ for all $t \geq 0$ and $i \neq j$,

2. $\sum_{j \neq i} q_{i j}(t)<\infty$ for all $t \geq 0$ and $i \in S$,

3. $q_{i}(t):=-q_{i i}(t)=\sum_{j \neq i} q_{i j}(t)$ for all $t \geq 0$ and $i \in S$, and

4. $q_{i}(t)$ is uniformly bounded, i.e. there exists a constant $M>0$ such that $q_{i}(t) \leq M$ for all $t \geq 0$ and $i \in S$,

then we call $Q(\cdot)$ a conservative inhomogeneous transition density matrix function on $S$. Furthermore, we say that $Q(\cdot)$ is continuous if $q_{i j}(\cdot), i, j \in S$, are all continuous.

Definition 2.2. Suppose that $Q(\cdot)$ is a continuous conservative inhomogeneous transition density matrix function. If there exists an inhomogeneous Markov process $X=\{X(t): t \geq 0\}$ with state space $S$, whose transition probability matrices

$$
P(s, t)=\left(p_{i j}(s, t)=\mathrm{P}(X(t)=j \mid X(s)=i)\right)_{i, j \in S, \quad t \geq s \geq 0,}
$$

satisfy the forward Kolmogorov equation, i.e.

$$
\frac{\partial P(s, t)}{\partial t}=P(s, t) Q(t), \quad \lim _{t \downarrow s} P(s, t)=I,
$$

or, equivalently,

$$
\lim _{t \downarrow s} \frac{\partial p_{i j}(s, t)}{\partial t}=q_{i j}(s) \text { for all } i, j \in S,
$$

then we call $X$ an inhomogeneous Markov chain with transition density $Q(\cdot)$. In (2.1), $I$ denotes the identity matrix. 
Theorem 2.1. For any continuous conservative inhomogeneous transition density matrix function $Q(\cdot)$ and any distribution $\pi(0)$, there exists a unique inhomogeneous Markov chain $\{X(t): t \geq 0\}$ with transition density $Q(\cdot)$ and initial distribution $\pi(0)$. Moreover, its transition probability matrices, $P(s, t)$, are continuous in both $s$ and $t$, and also satisfy the backward Kolmogorov equation, i.e.

$$
\frac{\partial P(s, t)}{\partial s}=-Q(s) P(s, t) .
$$

Moreover, $\pi(t)=\left(\pi_{i}(t)=\mathrm{P}(X(t)=i)\right)_{i \in S}$ is also continuously differentiable, and satisfies the Fokker-Planck equation, i.e.

$$
\frac{\mathrm{d} \pi(t)}{\mathrm{d} t}=\pi(t) Q(t)
$$

Proposition 2.1. For all $s \geq 0$, let $\tau_{s}=\inf \{t>0: X(t+s) \neq X(s)\}$. Then

$$
\begin{gathered}
\mathrm{P}\left(\tau_{s}>t \mid X(s)=i\right)=\exp \left[-\int_{s}^{s+t} q_{i}(u) \mathrm{d} u\right], \\
\mathrm{P}\left(X\left(s+\tau_{s}\right)=j \mid X(s)=i, \tau_{s}\right)=\frac{q_{i j}\left(s+\tau_{s}\right)}{q_{i}\left(s+\tau_{s}\right)} \text { for all } j \neq i, \\
\mathrm{P}\left(\tau_{s} \leq t, X\left(s+\tau_{s}\right)=j \mid X(s)=i\right)=\int_{s}^{s+t} q_{i j}(u) \exp \left[-\int_{s}^{u} q_{i}(v) \mathrm{d} v\right] \mathrm{d} u, \\
\mathrm{P}\left(X\left(s+\tau_{s}\right)=j \mid X(s)=i\right)=\int_{s}^{\infty} q_{i j}(u) \exp \left[-\int_{s}^{u} q_{i}(v) \mathrm{d} v\right] \mathrm{d} u .
\end{gathered}
$$

Suppose that $\pi_{i}(t)>0$ for all $i \in S$ and $t \geq 0$. For arbitrarily fixed $t$ and $s, t>s>0$, and any $u \in[s, t]$, let $Q^{-}(u)=\left(q_{i j}^{-}(u)\right)_{i, j \in S}$, where

$$
q_{i j}^{-}(u)=\frac{\pi_{j}(s+t-u)}{\pi_{i}(s+t-u)} q_{j i}(s+t-u), \quad i \neq j, \quad q_{i i}^{-}(u)=-\sum_{j \neq i} q_{i j}^{-}(u) .
$$

From the Fokker-Planck equation (2.3) and the backward Kolmogorov equation (2.2), we obtain the following result.

Proposition 2.2. For any $i, j \in S$ and $u$ and $v$ with $u \leq v \in[s, t]$, let

$$
p_{i j}^{-}(u, v)=\frac{\pi_{j}(s+t-v)}{\pi_{i}(s+t-u)} p_{j i}(s+t-v, s+t-u) .
$$

Then the matrix $P^{-}(u, v)=\left(p_{i j}^{-}(u, v)\right)_{i, j \in S}$ satisfies $\partial P^{-}(u, v) / \partial v=P^{-}(u, v) Q^{-}(v)$, i.e. $Q^{-}(\cdot)$ determines another family of inhomogeneous transition probability matrices, $P^{-}(u, v)$, which is the same as that of $\{X(s+t-u): s \leq u \leq t\}$, the time reverse of $\{X(u): s \leq u \leq t\}$.

\section{Reversibility and entropy production}

\subsection{Instantaneous reversibility and entropy production}

Definition 3.1. If, at time $s$, an inhomogeneous Markov chain $X=\{X(t): t \geq 0\}$ satisfies

$$
\lim _{t \downarrow s} \frac{\pi_{i}(s) p_{i j}(s, t)}{\pi_{j}(s) p_{j i}(s, t)}=1
$$


for every $i, j \in S$ such that $q_{i j}(s)>0$, then we say that the Markov chain $X$ is instantaneously reversible at time $s$. If $X$ is instantaneously reversible at any time, then we say that $X$ is instantaneously reversible.

We obtain the next result from l'Hôpital's rule.

Proposition 3.1. The Markov chain $X$ is instantaneously reversible at time $s$ if and only if $\pi_{i}(s) q_{i j}(s)=\pi_{j}(s) q_{j i}(s)$ for all $i, j \in S$, and $X$ is instantaneously reversible if and only if, for all $s, \pi_{i}(s) q_{i j}(s)=\pi_{j}(s) q_{j i}(s)$ for all $i, j \in S$.

This is just the detailed balance condition.

If $X$ is instantaneously reversible at time $s$, then obviously $\pi(s) Q(s)=0$.

Corollary 3.1. The Markov chain $X$ is instantaneously reversible if and only if each of the transition density matrices $Q(t)$ is reversible when it is regarded as the transition density matrix of a homogeneous Markov chain and their reversible distributions are the same.

Definition 3.2. As in the case of homogeneous Markov chains [26], the instantaneous entropy production rate, $e_{\mathrm{p}}(s)$, of $X$ can be defined from a physical point of view as

$$
e_{\mathrm{p}}(s)=\frac{1}{2} \sum_{i, j \in S}\left[\pi_{i}(s) q_{i j}(s)-\pi_{j}(s) q_{j i}(s)\right] \log \frac{\pi_{i}(s) q_{i j}(s)}{\pi_{j}(s) q_{j i}(s)} .
$$

It follows immediately that $e_{\mathrm{p}}(s)$ is nonnegative for all $s$, and that $e_{\mathrm{p}}(s)=0$ if and only if $\pi_{i}(s) q_{i j}(s)=\pi_{j}(s) q_{j i}(s)$ for all $i, j \in S$. This proves Theorem 1.1.

Corollary 3.2. The following statements are equivalent.

1. The Markov chain $X$ is instantaneously reversible.

2. It is in detailed balance: $\pi_{i}(s) q_{i j}(s)=\pi_{j}(s) q_{j i}(s)$ for all $s \geq 0$ and $i, j \in S$.

3. Its instantaneous entropy production rate vanishes at all times.

\subsection{Time-periodic inhomogeneous Markov chains and periodic reversibility}

Suppose that $Q(\cdot)$ is periodic with period $T>0$ and fix an $s \in[0, T)$. Then $\{X(k T+s): k=$ $0,1,2, \ldots\}$ is a homogeneous Markov chain with a denumerable state space.

Assumption 3.1. For all $s \in[0, T),\{X(k T+s): k=0,1,2, \ldots\}$ has a unique invariant distribution $\pi(s)$.

Obviously, for all $t>s, \theta(s, t)=\pi(s) P(s, t)$ is an invariant distribution of $\{X(k T+t): k=$ $0,1,2, \ldots\}$, so $\theta(s, t)=\pi(s) P(s, t)=\pi(t)$. That is, if $\pi(0)$ is the initial distribution of $X$, then the distribution of $X(t)$ is $\pi(t(\bmod T))$ for all $t$. Furthermore, for any $k \in \mathbb{Z}^{+}$, $\{X(k T+t): t \geq 0\}$ has the same distribution as $X=\{X(t): t \geq 0\}$, i.e. $X$ is periodically stationary. Therefore, we call $\{\pi(s): 0 \leq s<T\}$ the periodically invariant distribution of $X$.

From the strong law of large numbers for the homogeneous Markov chain $\{X(k T+s): k=$ $0,1,2, \ldots\}$, we obtain the following results.

Proposition 3.2. For any bounded function $f(i), i \in S$,

$$
\lim _{t \rightarrow \infty} \mathrm{E}\left|\frac{1}{t} \int_{S}^{s+t} f(X(u)) \mathrm{d} u-\frac{1}{T} \sum_{i \in S} \int_{0}^{T} \pi_{i}(u) f(i) \mathrm{d} u\right|^{p}=0, \quad p \geq 1 .
$$


Proof. By Jensen's inequality and the dominated convergence theorem,

$$
\begin{aligned}
\lim _{t \rightarrow \infty} \mathrm{E}\left|\frac{1}{t} \int_{s}^{s+t} f(X(u)) \mathrm{d} u-\frac{1}{T} \sum_{i \in S} \int_{0}^{T} \pi_{i}(u) f(i) \mathrm{d} u\right|^{p} \\
=\lim _{n \rightarrow \infty} \mathrm{E}\left|\frac{1}{n T} \int_{s}^{s+n T} f(X(u)) \mathrm{d} u-\frac{1}{T} \sum_{i \in S} \int_{0}^{T} \pi_{i}(u) f(i) \mathrm{d} u\right|^{p} \\
=\lim _{n \rightarrow \infty} \mathrm{E}\left|\frac{1}{n T} \sum_{i=1}^{n} \int_{s+(i-1) T}^{s+i T} f(X(u)) \mathrm{d} u-\frac{1}{T} \sum_{i \in S} \int_{0}^{T} \pi_{i}(u) f(i) \mathrm{d} u\right|^{p} \\
=\lim _{n \rightarrow \infty} \mathrm{E}\left|\frac{1}{T} \int_{s}^{s+T} \frac{1}{n} \sum_{i=1}^{n} f(X(u+(i-1) T)) \mathrm{d} u-\frac{1}{T} \int_{s}^{s+T} \sum_{i \in S} \pi_{i}(u) f(i) \mathrm{d} u\right|^{p} \\
\leq \frac{1}{T} \lim _{n \rightarrow \infty} \int_{s}^{s+T} \mathrm{E}\left|\frac{1}{n} \sum_{i=1}^{n} f(X(u+(i-1) T))-\sum_{i \in S} \pi_{i}(u) f(i)\right|^{p} \mathrm{~d} u \\
=\frac{1}{T} \int_{s}^{s+T} \lim _{n \rightarrow \infty} \mathrm{E}\left|\frac{1}{n} \sum_{i=1}^{n} f(X(u+(i-1) T))-\sum_{i \in S} \pi_{i}(u) f(i)\right|^{p} \mathrm{~d} u \\
=0 .
\end{aligned}
$$

We define the average entropy production rate of a time-periodic inhomogeneous Markov chain $X$ as $e_{\mathrm{p}}:=(1 / T) \int_{0}^{T} e_{\mathrm{p}}(t) \mathrm{d} t$. Thus, from the continuity of $e_{\mathrm{p}}(\cdot), e_{\mathrm{p}}=0$ is equivalent to $e_{\mathrm{p}}(t)=0$ for all $t$. So $e_{\mathrm{p}}=0$ if and only if $X$ is instantaneously reversible.

Let $T_{0}=0$ and $T_{k}=\inf \left\{t>T_{k-1}: X(t) \neq X\left(T_{k-1}\right)\right\}, k \geq 1$. Then, from the construction in [30], we know that $\left\{\left(T_{k}(\bmod T), X\left(T_{k}\right)\right): k=0,1,2, \ldots\right\}$ is a two-dimensional homogeneous Markov chain whose transition probability density, $p(s, t ; i, j)$, is

$$
p(s, t ; i, j)=\left\{\begin{aligned}
q_{i j}(t) \sum_{k=0}^{\infty} \mathrm{e}^{-\int_{s}^{k T+t} q_{i}(u) \mathrm{d} u} & =q_{i j}(t) \sum_{k=0}^{\infty} \mathrm{e}^{-\int_{s}^{t} q_{i}(u) \mathrm{d} u}\left(\mathrm{e}^{-\int_{0}^{T} q_{i}(u) \mathrm{d} u}\right)^{k} \\
& =q_{i j}(t) \mathrm{e}^{-\int_{s}^{t} q_{i}(u) \mathrm{d} u}\left(1-\mathrm{e}^{-\int_{0}^{T} q_{i}(u) \mathrm{d} u}\right)^{-1}, \quad t \geq s, \\
q_{i j}(t) \sum_{k=1}^{\infty} \mathrm{e}^{-\int_{s}^{k T+t} q_{i}(u) \mathrm{d} u} & =q_{i j}(t) \sum_{k=0}^{\infty} \mathrm{e}^{-\int_{s}^{t+T} q_{i}(u) \mathrm{d} u}\left(\mathrm{e}^{-\int_{0}^{T} q_{i}(u) \mathrm{d} u}\right)^{k} \\
& =q_{i j}(t) \mathrm{e}^{-\int_{s}^{t+T} q_{i}(u) \mathrm{d} u}\left(1-\mathrm{e}^{-\int_{0}^{T} q_{i}(u) \mathrm{d} u}\right)^{-1}, \quad t<s,
\end{aligned}\right.
$$

with $j \neq i$. We denote its invariant distribution by $\hat{\pi}_{i}(s)$.

Applying Corollary 3.1, we then obtain the following lemma.

Lemma 3.1. Let $Z=\int_{0}^{T} \sum_{i} \pi_{i} q_{i}(s) \mathrm{d} s$. If $X$ is instantaneously reversible, then $\hat{\pi}_{i}(s)=$ $\pi_{i} q_{i}(s) / Z$.

Example 3.1. (Time-periodic birth-death Markov chains.) Suppose that $S=\{1,2, \ldots, N\}$ with the $N$ points lying on a ring, with $i$ and $i+1$ and 1 and $N$ being neighbors. Also assume that $q_{i, i+1}(t)+q_{i, i-1}=q_{i}(t)$ for all $i \in S$ and $t \geq 0$. Then $X$ is a time-periodic birth-death Markov chain on a ring. Such a Markov chain can be regarded as an approximation of the Brownian motor model (1.1). 
Theorem 3.1. Let

$$
f(i, j)= \begin{cases}1, & j=i+1 \text { or } i=N \text { and } j=1, \\ -1, & j=i-1 \text { or } i=1 \text { and } j=N, \\ 0, & j=i .\end{cases}
$$

Then we can define the rotation number of the birth-death chain $X=\{X(t): t \geq 0\}$ as

$$
\begin{aligned}
\text { Rot } & :=\lim _{t \rightarrow \infty} \frac{1}{t} \sum_{k=0}^{n_{t}-1} f\left(X\left(T_{k}\right), X\left(T_{k+1}\right)\right) \\
& =\left(\lim _{t \rightarrow \infty} \frac{n_{t}}{t}\right) \sum_{i=1}^{N} \int_{0}^{T} \int_{0}^{T} \hat{\pi}_{i}(s)(p(s, t ; i, i+1)-p(s, t ; i, i-1)) \mathrm{d} s \mathrm{~d} t,
\end{aligned}
$$

where $n_{t}$ denotes the number of times that $X$ jumps in $[0, t]$. Moreover, if the birth-death chain $X$ is instantaneously reversible, then the rotation number vanishes.

Proof. First, we use the strong law of large numbers for the two-dimensional homogeneous Markov chain $\left\{\left(T_{k}(\bmod T), X\left(T_{k}\right)\right): k=0,1,2, \ldots\right\}$, to conclude that

$\lim _{t \rightarrow \infty} \frac{1}{n_{t}} \sum_{k=0}^{n_{t}-1} f\left(X\left(T_{k}\right), X\left(T_{k+1}\right)\right)=\sum_{i=1}^{N} \int_{0}^{T} \int_{0}^{T} \hat{\pi}_{i}(s)(p(s, t ; i, i+1)-p(s, t ; i, i-1)) \mathrm{d} s \mathrm{~d} t$.

Second, by the subadditive ergodic theorem in [27, p. 231], as $k$ tends to $\infty, n_{k T} / k T$ converges almost everywhere to a random variable with expectation $\mathrm{E}\left(n_{T}\right) / T$. Then, by the monotonicity of $n_{t}, \lim _{t \rightarrow \infty} n_{t} / t=\lim _{k \rightarrow \infty} n_{k T} / k T$.

If the birth-death chain $X$ is instantaneously reversible, then, by Lemma 3.1,

$$
\begin{aligned}
& \sum_{i=1}^{N} \int_{0}^{T} \int_{0}^{T} \hat{\pi}_{i}(s)(p(s, t ; i, i+1)-p(s, t ; i, i-1)) \mathrm{d} s \mathrm{~d} t \\
& =\sum_{i=1}^{N} \frac{\int_{0}^{T} q_{i}(s) \mathrm{d} s \int_{s}^{s+T} D_{i}(t) \mathrm{e}^{-\int_{s}^{t} q_{i}(u) \mathrm{d} u} \mathrm{~d} t}{Z_{i}} \\
& =\sum_{i=1}^{N} \frac{1}{Z_{i}}\left\{\int_{0}^{T} D_{i}(t) \mathrm{d} t \int_{0}^{t} \mathrm{e}^{-\int_{s}^{t} q_{i}(u) \mathrm{d} u} q_{i}(s) \mathrm{d} s+\int_{T}^{2 T} D_{i}(t) \mathrm{d} t \int_{t-T}^{T} \mathrm{e}^{-\int_{s}^{t} q_{i}(u) \mathrm{d} u} q_{i}(s) \mathrm{d} s\right\} \\
& =\sum_{i=1}^{N} \frac{1}{Z_{i}}\left\{\int_{0}^{T} D_{i}(t)\left(1-\mathrm{e}^{-\int_{0}^{t} q_{i}(u) \mathrm{d} u}\right) \mathrm{d} t+\int_{T}^{2 T} D_{i}(t)\left(\mathrm{e}^{-\int_{T}^{t} q_{i}(u) \mathrm{d} u}-\mathrm{e}^{-\int_{t-T}^{t} q_{i}(u) \mathrm{d} u}\right) \mathrm{d} t\right\} \\
& =\sum_{i=1}^{N} \frac{1}{Z_{i}}\left\{\int_{0}^{T} D_{i}(t)\left(1-\mathrm{e}^{-\int_{0}^{t} q_{i}(u) \mathrm{d} u}\right) \mathrm{d} t+\int_{0}^{T} D_{i}(t)\left(\mathrm{e}^{-\int_{0}^{t} q_{i}(u) \mathrm{d} u}-\mathrm{e}^{-\int_{0}^{T} q_{i}(u) \mathrm{d} u}\right) \mathrm{d} t\right\} \\
& =\frac{1}{Z} \sum_{i=1}^{N} \int_{0}^{T} D_{i}(t) \mathrm{d} t \\
& =0 \text {, }
\end{aligned}
$$

where $D_{i}(t)=\pi_{i} q_{i, i+1}(t)-\pi_{i} q_{i, i-1}(t)$ and $Z_{i}=Z\left(1-\mathrm{e}^{-\int_{0}^{T} q_{i}(u) \mathrm{d} u}\right)$. 
Remark 3.1. If $Q(t) \equiv Q$, i.e. $\{X(t): t \geq 0\}$ is a homogeneous, irreducible Markov chain with invariant distribution $\pi$, then, by [15, Lemma 2.2.1], $\lim _{t \rightarrow \infty} n_{t} / t=-\sum_{i} \pi_{i} q_{i i}$.

In the time-periodic inhomogeneous case, although we can prove that $n_{t} / t$ converges to a limit random variable almost everywhere, we cannot prove that the limit is almost surely a constant, and express it in terms of the elements of $Q(\cdot)$ and $\pi(\cdot)$.

In the last theorem, Rot/ $N$ is just the average number of occurrences of the circle $1 \rightarrow 2 \rightarrow$ $3 \rightarrow \cdots \rightarrow N \rightarrow 1$ along almost all the trajectories of $X$.

From the Fokker-Planck equation (2.3), we know that $\int_{0}^{T} \pi(t) Q(t) \mathrm{d} t=0$, so the probability flux from $i$ to $i+1$,

$$
C_{i}:=\frac{1}{T} \int_{0}^{T}\left[\pi_{i}(t) q_{i, i+1}(t)-\pi_{i+1}(t) q_{i+1, i}(t)\right] \mathrm{d} t,
$$

is independent of $i$. In the case where the birth-death chain $X$ is instantaneously reversible, $C_{i}=0$ for all $i$, i.e. all the probability fluxes vanish.

Definition 3.3. If, for some $s \in[0, T)$, the time-periodic inhomogeneous Markov chain $X$ is such that $\{X(t): s \leq t \leq n T+s\}$ and $\{X(n T+2 s-t): s \leq t \leq n T+s\}$ have the same distribution for all $n \in \mathbb{Z}^{+}$, then we say that the Markov chain $X$ has periodic reversibility with index $s$.

If the Markov chain $X$ is such that $\{X(t): s \leq t \leq n T+s\}$ and $\{X(n T+2 s-t): s \leq t \leq$ $n T+s\}$ have the same distribution for all $s \in[0, T)$ and all $n \in \mathbb{Z}^{+}$, then we say that $X$ has complete periodic reversibility.

Here, we only consider periodic reversibility with index $s$. Some surprising results about complete periodic reversibility will be presented in Section 5 .

Under Assumption 3.1, if $X$ has periodic reversibility with index $s$, then $X$ is periodically stationary.

Proposition 3.3. The following statements are equivalent.

1. The Markov chain $X$ has periodic reversibility with index $s$.

2. $\{X(t): s \leq t \leq T+s\}$ and $\{X(T+2 s-t): s \leq t \leq T+s\}$ have the same distribution, i.e.

$$
\begin{aligned}
& \pi_{i_{0}}(s) p_{i_{0} i_{1}}\left(t_{0}, t_{1}\right) \cdots p_{i_{n-1} i_{n}}\left(t_{n-1}, t_{n}\right) \\
& \quad=\pi_{i_{n}}(s) p_{i_{n} i_{n-1}}\left(T+2 s-t_{n}, T+2 s-t_{n-1}\right) \cdots p_{i_{1} i_{0}}\left(T+2 s-t_{1}, T+2 s-t_{0}\right)
\end{aligned}
$$

for all $n \in \mathbb{Z}^{+}, t_{i}$ with $t_{0}=s<t_{1}<\cdots<t_{n}=T+s$, and $i_{0}, \ldots, i_{n} \in S$.

3. For all $t_{1}$ and $t_{2}$ with $s \leq t_{1}<t_{2} \leq s+T$, and $i, j \in S$,

$$
\pi_{i}\left(t_{1}\right) p_{i j}\left(t_{1}, t_{2}\right)=\pi_{j}\left(T+2 s-t_{2}\right) p_{j i}\left(T+2 s-t_{2}, T+2 s-t_{1}\right) .
$$

4. For all $t$ with $s \leq t \leq s+T$, and $i, j \in S$,

$$
\begin{array}{ccc}
\pi_{i}(t)=\pi_{i}(T+2 s-t), & \\
\pi_{i}(t) q_{i j}(t)=\pi_{j}(T+2 s-t) q_{j i}(T+2 s-t), & i \neq j, \\
\pi_{i}(t) q_{i i}(t)=-\sum_{k \neq i} \pi_{k}(T+2 s-t) q_{k i}(T+2 s-t), & i=j .
\end{array}
$$

This is the detailed balance condition. 
Proof. That statement 1 implies statement 2 follows directly from the Markov property of $X$. That statement 2 implies statement 3 follows from summing over $i_{0}, i_{3}, \ldots, i_{n}$ in (3.1) for arbitrarily fixed $i_{1}, i_{2}, t_{1}$, and $t_{2}$. To obtain the first equation in statement 4 , sum both sides of (3.2) over $j$. To obtain the other two equations in statement 4 , differentiate both sides of (3.2) with respect to $t_{2}$, use the forward and backward Kolmogorov equations (2.1) and (2.2) and the Fokker-Planck equation (2.3), and then let $t_{2} \downarrow t_{1}$. Thus, statement 3 implies statement 4 . That statement 4 implies statement 1 follows from Proposition 2.2, since $q_{i j}^{-}(t)=q_{i j}(t)$ for all $t \in[s, s+T]$.

Although we cannot calculate $\hat{\pi}_{i}(s)$ explicitly, we do have the following theorem for the birth-death Markov chain in Example 3.1.

Theorem 3.2. If the time-periodic birth-death Markov chain $X$ on a ring has periodic reversibility with some index $s$, then the rotation number Rot vanishes with probability 1.

Proof. We can prove only the case where $s=0$.

By assumption, $\{X(t): 0 \leq t \leq n T\}$ and its time reverse $X_{(n)}^{-}=\{X(n T-t): 0 \leq t \leq n T\}$ have the same distribution for each $n \in \mathbb{Z}^{+}$. We can define the rotation number, $\operatorname{Rot}^{(n)}$, of the birth-death Markov chain $X_{(n)}^{-}$, so $R o t \stackrel{\mathrm{D}}{=} \operatorname{Rot}^{(n)} \stackrel{\mathrm{D}}{=}-\operatorname{Rot}$ for all $n$, where $\stackrel{\text { D }}{=}$ ' denotes equality in distribution. On the other hand, Rot is almost everywhere a constant. Therefore, this constant must be 0 , i.e. the rotation number Rot vanishes with probability 1 .

\section{Measure-theoretic definition of instantaneous entropy production}

Theorem 4.1. The instantaneous entropy production rate, $e_{\mathrm{p}}(s)$ (see Definition 3.2), of the inhomogeneous Markov chain $\{X(t): t \geq 0\}$ can also be measure-theoretically defined as

$$
e_{\mathrm{p}}(s)=\lim _{t \downarrow s} \frac{1}{t-s} H\left(\mathrm{P}_{[s, t]}, \mathrm{P}_{[s, t]}^{-}\right)
$$

where $\mathrm{P}_{[s, t]}$ is the distribution of $\{X(u): s \leq u \leq t\}, \mathrm{P}_{[s, t]}^{-}$is the distribution of $\{X(s+t-$ $u): s \leq u \leq t\}$, and $H\left(\mathrm{P}_{[s, t]}, \mathrm{P}_{[s, t]}^{-}\right)$is the relative entropy of $\mathrm{P}_{[s, t]}$ with respect to $\mathrm{P}_{[s, t]}^{-}$.

Before proving the theorem, we first prove two lemmas.

Lemma 4.1. For arbitrarily fixed $t$ and $s, t>s>0$, and any $i \in S$ and $u$ and $v, u \leq v \in[s, t]$,

$$
\pi_{i}(v) \exp \left[\int_{u}^{v} q_{i i}^{-}(s+t-w) \mathrm{d} w\right]=\pi_{i}(u) \exp \left[\int_{u}^{v} q_{i i}(w) \mathrm{d} w\right] .
$$

Proof. It is easy to check that both sides satisfy the same ordinary differential equation,

$$
\frac{\mathrm{d} f(v)}{\mathrm{d} v}=q_{i i}(v) f(v), \quad f(u)=\pi_{i}(u) .
$$

The desired result follows from the uniqueness of its solution.

Denote by $n_{t}$ the number of times that $X$ jumps in $[s, t]$. Let $T_{0}=s, T_{1}=\inf \{t>s: X(t) \neq$ $X(s)\}, T_{k}=\inf \left\{t>T_{k-1}: X(t) \neq X\left(T_{k-1}\right)\right\}$, and $T_{n_{t}+1}=t$. Then, for all $i_{0}, \ldots, i_{n} \in S$ satisfying $i_{k} \neq i_{k+1}, 0 \leq k<n$, we can define

$$
A_{i_{0} \cdots i_{n}}(t)=\left\{\omega \in \Omega: n_{t}(\omega)=n, X(s)=i_{0}, X\left(T_{k}(\omega)\right)=i_{k}, k=1, \ldots, n\right\} .
$$


Lemma 4.2. If $Q(\cdot)$ satisfies the condition

$$
q_{i j}(t)>0 \text { for some } t \quad \Longleftrightarrow \quad q_{j i}(t)>0 \text { for all } t,
$$

then $\mathrm{P}_{[s, t]}$ and $\mathrm{P}_{[s, t]}^{-}$are absolutely continuous with respect to each other. The Radon-Nikodým derivative is almost everywhere

$$
\left.\frac{\mathrm{dP}_{[s, t]}}{\mathrm{dP}_{[s, t]}^{-}}\right|_{A_{i_{0} \cdots i_{n}}(t)}=\frac{\pi_{i_{0}}(s) \prod_{k=0}^{n-1} q_{i_{k} i_{k+1}}\left(T_{k+1}\right) \exp \left[-\sum_{k=0}^{n} \int_{T_{k}}^{T_{k+1}} q_{i_{k}}(u) \mathrm{d} u\right]}{\pi_{i_{n}}(s) \prod_{k=0}^{n-1} q_{i_{n-k} i_{n-k+1}}\left(s+t-T_{n-k}\right) \exp \left[-\sum_{k=0}^{n} \int_{s+t-T_{k+1}}^{s+t-T_{k}} q_{i_{k}}(u) \mathrm{d} u\right]} .
$$

Proof. For all $i_{0}, \ldots, i_{n} \in S$ and $t_{0}, \ldots, t_{n+1}$ satisfying $i_{k} \neq i_{k+1}$ for $0 \leq k<n$ and $t_{0}=s<t_{1}<\cdots<t_{n+1}=t$, we can choose a sufficiently small $\delta t_{k}$ and define

$$
A=\left\{\omega \in \Omega: n_{t}(\omega)=n, X(s)=i_{0}, t_{k}<T_{k}<t_{k}+\delta t_{k}, X\left(T_{k}(\omega)\right)=i_{k}, k=1, \ldots, n\right\} .
$$

From Proposition 2.1 and Lemma 4.1, we have

$$
\begin{array}{r}
\mathrm{P}_{[s, t]}(A)=\int_{t_{1}}^{t_{1}+\delta t_{1}} \mathrm{~d} s_{1} \cdots \int_{t_{n}}^{t_{n}+\delta t_{n}} \mathrm{~d} s_{n} \pi_{i_{0}}(s) q_{i_{0} i_{1}}\left(s_{1}\right) \cdots q_{i_{n-1} i_{n}}\left(s_{n}\right) \\
\times \exp \left[-\sum_{k=0}^{n} \int_{s_{k}}^{s_{k+1}} q_{i_{k}}(u) \mathrm{d} u\right]
\end{array}
$$

and

$$
\begin{array}{r}
\mathrm{P}_{[s, t]}^{-}(A)=\int_{t_{1}}^{t_{1}+\delta t_{1}} \mathrm{~d} s_{1} \cdots \int_{t_{n}}^{t_{n}+\delta t_{n}} \mathrm{~d} s_{n} \pi_{i_{0}}^{-}(s) q_{i_{0} i_{1}}^{-}\left(s_{1}\right) \cdots q_{i_{n-1} i_{n}}^{-}\left(s_{n}\right) \\
\times \exp \left[-\sum_{k=0}^{n} \int_{s_{k}}^{s_{k+1}} q_{i_{k}}^{-}(u) \mathrm{d} u\right] \\
=\int_{t_{1}}^{t_{1}+\delta t_{1}} \mathrm{~d} s_{1} \cdots \int_{t_{n}}^{t_{n}+\delta t_{n}} \mathrm{~d} s_{n} \pi_{i_{n}}(s) q_{i_{n} i_{n-1}}\left(s+t-s_{n}\right) \cdots q_{i_{1} i_{0}}\left(s+t-s_{1}\right) \\
\times \exp \left[-\sum_{k=0}^{n} \int_{s+t-s_{k+1}}^{s+t-s_{k}} q_{i_{k}}(u) \mathrm{d} u\right]
\end{array}
$$

with $s_{0}=s$ and $s_{n+1}=t$. Thus,

$$
\begin{aligned}
\mathrm{P}_{[s, t]}(A)=0 & \Longleftrightarrow \pi_{i_{0}}(s) q_{i_{0} i_{1}}\left(s_{1}\right) \cdots q_{i_{n-1} i_{n}}\left(s_{n}\right)=0 \\
& \Longleftrightarrow \pi_{i_{n}}(s) q_{i_{n} i_{n-1}}\left(s+t-s_{n}\right) \cdots q_{i_{1} i_{0}}\left(s+t-s_{1}\right)=0 \\
& \Longleftrightarrow P_{[s, t]}^{-}(A)=0 .
\end{aligned}
$$

The desired result follows because $\sigma\left(X_{u}: s \leq u \leq t\right)$ is generated by such sets $A$.

Proof of Theorem 4.1. We have

$$
\begin{aligned}
H\left(\mathrm{P}_{[s, t]}, \mathrm{P}_{[s, t]}^{-}\right) & =\mathrm{E}\left(\log \frac{\mathrm{dP}_{[s, t]}}{\mathrm{dP}_{[s, t]}^{-}}\right) \\
& =\sum_{n=0}^{\infty} \mathrm{E}\left(\log \frac{\mathrm{dP}_{[s, t]}}{\mathrm{dP}_{[s, t]}^{-}} \mid n_{t}=n\right) \mathrm{P}\left(n_{t}=n\right),
\end{aligned}
$$


and

$$
\begin{aligned}
& \mathrm{E}\left(\log \frac{\mathrm{dP}_{[s, t]}}{\mathrm{dP}_{[s, t]}^{-}} \mid n_{t}=n\right) \\
& =\mathrm{E}\left(\log \frac{\pi_{X\left(T_{0}\right)}(s) \prod_{k=0}^{n-1} q_{X\left(T_{k}\right) X\left(T_{k+1}\right)}\left(T_{k+1}\right) \exp \left[-\sum_{k=0}^{n} \int_{T_{k}}^{T_{k+1}} q_{X\left(T_{k}\right)}(u) \mathrm{d} u\right]}{\pi_{X\left(T_{n}\right)}(s) \prod_{k=0}^{n-1} q_{X\left(T_{k+1}\right) X\left(T_{k}\right)}\left(s+t-T_{k+1}\right) \exp \left[-\sum_{k=0}^{n} \int_{s+t-T_{k+1}}^{s+t-T_{X}} q_{X\left(T_{k}\right)}(u) \mathrm{d} u\right]} \mid n_{t}=n\right) \\
& =\sum_{k=0}^{n-1} \mathrm{E}\left(\log \frac{q_{X\left(T_{k}\right) X\left(T_{k+1}\right)}\left(T_{k+1}\right)}{q_{X\left(T_{k+1}\right) X\left(T_{k}\right)}\left(s+t-T_{k+1}\right)} \mid n_{t}=n\right)+\mathrm{E}\left(\log \frac{\pi_{X\left(T_{0}\right)}(s)}{\pi_{X\left(T_{n}\right)}(s)} \mid n_{t}=n\right) \\
& \quad+\mathrm{E}\left(\sum_{k=0}^{n} \int_{s+t-T_{k+1}}^{s+t-T_{k}} q_{X\left(T_{k}\right)}(u) \mathrm{d} u-\sum_{k=0}^{n} \int_{T_{k}}^{T_{k+1}} q_{X\left(T_{k}\right)}(u) \mathrm{d} u \mid n_{t}=n\right) .
\end{aligned}
$$

Let

$$
\begin{aligned}
& C_{1}(s)=\max \left\{\left|\log \frac{q_{i j}(u)}{q_{j i}(v)}\right|: i, j \in S, q_{i j}(s)>0, s \leq u, v \leq s+1\right\}, \\
& C_{2}(s)=\max _{i, j \in S}\left|\log \frac{\pi_{i}(s)}{\pi_{j}(s)}\right|, \\
& C_{3}(s)=\max \left\{q_{i}(u): i \in S, s \leq u \leq s+1\right\} .
\end{aligned}
$$

For $n \geq 2$, by (4.1) we have

$$
\left|\mathrm{E}\left(\log \frac{\mathrm{dP}_{[s, t]}}{\mathrm{dP}_{[s, t]}^{-}} \mid n_{t}=n\right)\right| \leq C_{1}(s) n+C_{2}(s)+C_{3}(s)(n+1)(t-s) .
$$

Notice that

$$
\begin{aligned}
\mathrm{P}\left(A_{i_{0} \cdots i_{n}}(t)\right)=\int \cdots \int_{s<s_{1}<\cdots<s_{n}<t} \mathrm{~d} s_{1} \cdots \mathrm{d} s_{n} \\
\quad \times \pi_{i_{0}}(s) q_{i_{0} i_{1}}\left(s_{1}\right) \cdots q_{i_{n-1} i_{n}}\left(s_{n}\right) \exp \left[-\sum_{k=0}^{n} \int_{s_{k}}^{s_{k+1}} q_{i_{k}}(u) \mathrm{d} u\right] \\
\leq \int \cdots \int_{s<s_{1}<\cdots<s_{n}<t} \mathrm{~d} s_{1} \cdots \mathrm{d} s_{n} \\
\times \pi_{i_{0}}(s) q_{i_{0} i_{1}}\left(s_{1}\right) \cdots q_{i_{n-1} i_{n}}\left(s_{n}\right) .
\end{aligned}
$$

Therefore,

$$
\begin{aligned}
\mathrm{P}\left(n_{t}=n\right) & =\sum_{\substack{i_{0}, \ldots, i_{n} \\
i_{k} \neq i_{k+1}}} \mathrm{P}\left(A_{i_{0} \cdots i_{n}}(t)\right) \\
& \leq \int \cdots \int_{s<s_{1}<\cdots<s_{n}<t} \mathrm{~d} s_{1} \cdots \mathrm{d} s_{n} \sum_{\substack{i_{0}, \ldots, i_{n} \\
i_{k} \neq i_{k+1}}} \pi_{i_{0}}(s) q_{i_{0} i_{1}}\left(s_{1}\right) \cdots q_{i_{n-1} i_{n}}\left(s_{n}\right) \\
& \leq \frac{\left(C_{3}(s)(t-s)\right)^{n}}{n !} .
\end{aligned}
$$


Hence, by (4.2) and (4.3),

$$
\sum_{n=2}^{\infty} \mathrm{E}\left(\log \frac{\mathrm{dP}_{[s, t]}}{\mathrm{dP}_{[s, t]}^{-}} \mid n_{t}=n\right) \mathrm{P}\left(n_{t}=n\right)=O\left((t-s)^{2}\right) \quad \text { as } t \downarrow s .
$$

Then, from

$$
\begin{aligned}
& H\left(\mathrm{P}_{[s, t]}, \mathrm{P}_{[s, t]}^{-}\right)=\mathrm{E}\left(\log \frac{\mathrm{dP}_{[s, t]}}{\mathrm{dP}_{[s, t]}^{-}} \mid n_{t}=1\right) \mathrm{P}\left(n_{t}=1\right)+O\left((t-s)^{2}\right) \\
& =\left\{\mathrm{E}\left(\log \frac{\pi_{X(s)}(s) q_{X\left(T_{0}\right) X\left(T_{1}\right)}\left(T_{1}\right)}{\pi_{X(t)}(s) q_{X\left(T_{1}\right) X\left(T_{0}\right)}\left(s+t-T_{1}\right)} \mid n_{t}=1\right)\right. \\
& +\mathrm{E}\left(\int_{s+t-T_{1}}^{t} q_{X\left(T_{0}\right)}(u) \mathrm{d} u-\int_{s}^{T_{1}} q_{X\left(T_{0}\right)}(u) \mathrm{d} u \mid n_{t}=1\right) \\
& \left.+\mathrm{E}\left(\int_{s}^{s+t-T_{1}} q_{X\left(T_{1}\right)}(u) \mathrm{d} u-\int_{T_{1}}^{t} q_{X\left(T_{1}\right)}(u) \mathrm{d} u \mid n_{t}=1\right)\right\} \\
& \times \mathrm{P}\left(n_{t}=1\right)+O\left((t-s)^{2}\right) \\
& =\mathrm{E}\left(\log \frac{\pi_{X(s)}(s) q_{X\left(T_{0}\right) X\left(T_{1}\right)}\left(T_{1}\right)}{\pi_{X(t)}(s) q_{X\left(T_{1}\right) X\left(T_{0}\right)}\left(s+t-T_{1}\right)} \mid n_{t}=1\right) \\
& \times \mathrm{P}\left(n_{t}=1\right)+O\left((t-s)^{2}\right) \\
& =\sum_{i \neq j} \mathrm{E}\left(\log \frac{\pi_{i}(s) q_{i j}\left(T_{1}\right)}{\pi_{j}(s) q_{j i}\left(s+t-T_{1}\right)} \mid n_{t}=1, X(s)=i, X(t)=j\right) \\
& \times \mathrm{P}\left(n_{t}=1, X(s)=i, X(t)=j\right)+O\left((t-s)^{2}\right) \\
& =\sum_{i \neq j} \mathrm{E}\left(\log \frac{\pi_{i}(s) q_{i j}\left(T_{1}\right)}{\pi_{j}(s) q_{j i}\left(s+t-T_{1}\right)} \mid n_{t}=1, X(s)=i, X(t)=j\right) \\
& \times \int_{s}^{t} \pi_{i}(s) q_{i j}(u) \exp \left[-\int_{s}^{u} q_{i}(v) \mathrm{d} v-\int_{u}^{t} q_{j}(v) \mathrm{d} v\right] \mathrm{d} u \\
& +O\left((t-s)^{2}\right)
\end{aligned}
$$

we find that

$$
\begin{aligned}
e_{\mathrm{p}}(s) & =\lim _{t \downarrow s} \frac{1}{t-s} H\left(\mathrm{P}_{[s, t]}, \mathrm{P}_{[s, t]}^{-}\right) \\
& =\sum_{i, j \in S} \pi_{i}(s) q_{i j}(s) \log \frac{\pi_{i}(s) q_{i j}(s)}{\pi_{j}(s) q_{j i}(s)} \\
& =\frac{1}{2} \sum_{i, j \in S}\left[\pi_{i}(s) q_{i j}(s)-\pi_{j} q_{j i}(s)\right] \log \frac{\pi_{i}(s) q_{i j}(s)}{\pi_{j}(s) q_{j i}(s)} .
\end{aligned}
$$

\section{Remarks and examples}

\subsection{On complete periodic reversibility}

Apart from instantaneous reversibility, we have also investigated several other definitions of reversibility of inhomogeneous Markov processes, e.g. complete periodic reversibility, in [9]. 
Disappointingly, however, when a continuous-time Markov chain has complete periodic reversibility, it turns out to be homogeneous.

The next lemma is [6, Theorem 1.4].

Lemma 5.1. The transition probability matrices $P(s, t)$ are nondegenerate.

Proposition 5.1. Suppose that $Q(\cdot)$ is periodic with period $T$. If, for all $i_{0}, \ldots, i_{n} \in S$ and $t_{i}$ with $t=t_{0}<\cdots<t_{n}=t+T$, we have

$$
\begin{aligned}
& \pi_{i_{0}}(t) p_{i_{0} i_{1}}\left(t, t_{1}\right) \cdots p_{i_{n-1} i_{n}}\left(t_{n-1}, t+T\right) \\
& \quad=\pi_{i_{n}}(t) p_{i_{n} i_{n-1}}\left(t, T+2 t-t_{n-1}\right) \cdots p_{i_{1} i_{0}}\left(T+2 t-t_{1}, T+t\right),
\end{aligned}
$$

then $Q(t)$ does not change with the parameter $t$, i.e. $Q(\cdot)$ is homogeneous.

Proof. First, by summing over $i_{0}, i_{2}, \ldots, i_{n}$, we obtain $\pi_{i_{1}}\left(t_{1}\right)=\pi_{i_{1}}\left(T+2 t-t_{1}\right)$, so $\pi(s)$ does not change with the parameter $s$, because of the arbitrariness of $t_{1}$ and $t$. Second, by summing over $i_{0}, i_{3}, \ldots, i_{n}$, we obtain

$$
\pi_{i_{1}} p_{i_{1} i_{2}}\left(t_{1}, t_{2}\right)=\pi_{i_{1}} p_{i_{1} i_{2}}\left(T+2 t-t_{2}, T+2 t-t_{1}\right) .
$$

By the arbitrariness of $t, t_{1}$, and $t_{2}, P(s, s+u)$ depends only on $u$. Finally, we obtain the desired result by differentiating $P(s, s+u)$ with respect to $u$.

Remark 5.1. We must now emphasize that the complete periodic reversibility of [9] is meaningful for the discrete-time case. A discrete-time Markov chain with complete periodic reversibility degenerates to a homogeneous one when the period $T$ is odd; however, to the authors' surprise, it does not always degenerate to a homogeneous one when $T$ is even.

Here we use the notation of [9].

Example 5.1. There exist inhomogeneous examples which are completely periodically reversible. Let $T=2$ and

$$
P(0,1)=\left(\begin{array}{cc}
1-a & a \\
b & 1-b
\end{array}\right), \quad P(1,2)=\left(\begin{array}{cc}
1-c & c \\
d & 1-d
\end{array}\right) .
$$

This example is completely periodically reversible if and only if $c d(1-a-b)=a b(1-c-d)$.

Example 5.2. Let $T=2$ and

$$
P(0,1)=\left(\begin{array}{ccc}
0 & \frac{1}{3} & \frac{2}{3} \\
\frac{1}{3} & 0 & \frac{2}{3} \\
\frac{1}{2} & \frac{1}{2} & 0
\end{array}\right), \quad P(1,2)=\left(\begin{array}{ccc}
0 & \frac{1}{2} & \frac{1}{2} \\
\frac{1}{2} & 0 & \frac{1}{2} \\
\frac{1}{2} & \frac{1}{2} & 0
\end{array}\right) .
$$

This example is completely periodically reversible.

Theorem 5.1. Suppose that a periodically inhomogeneous Markov chain $\xi=\left\{\xi_{n}: n=\right.$ $0,1, \ldots\}$ with denumerable state space $S$ has complete periodic reversibility, and let

$$
\begin{aligned}
& P_{k}=\left\{p_{i j}(n T+k, n T+k+1):\right. \\
& p_{i j}(n T+K, n T+k+1)=\mathrm{P}\left(\xi_{n T+k+1}=j \mid \begin{array}{l}
j \\
\left.\xi_{n T+k}=i\right), \\
i, j, \in S, n=0,1, \ldots\}
\end{array}\right.
\end{aligned}
$$

for $k=0, \ldots, T-1$. If $T$ is odd then $P_{0}=\cdots=P_{T-1}$, i.e. $\xi$ is a homogeneous reversible Markov chain. If $T$ is even then $P_{0}=P_{2}=\cdots=P_{T-2}$ and $P_{1}=P_{3}=\cdots=P_{T-1}$. 
Proof. Recall that the periodically inhomogeneous Markov chain $\xi$ has complete periodic reversibility if and only if $\left(\xi_{k}, \xi_{k+1}, \ldots, \xi_{k+T}\right)$ and $\left(\xi_{k+T}, \xi_{k+T-1}, \ldots, \xi_{k}\right)$ have the same distribution for all $k=0, \ldots, T-1$, i.e. for any $k=0, \ldots, T-1$ and $i_{0}, \ldots, i_{T} \in S$,

$$
\begin{aligned}
& \pi_{i_{0}}^{k} p_{i_{0} i_{1}}(k, k+1) p_{i_{1} i_{2}}(k+1, k+2) \cdots p_{i_{T-1} i_{T}}(k+T-1, k+T) \\
& =\pi_{i_{T}}^{k} p_{i_{T} i_{T-1}}(k, k+1) p_{i_{T-1} i_{T-2}}(k+1, k+2) \cdots p_{i_{1} i_{0}}(k-1, k),
\end{aligned}
$$

where $\Pi_{k}=\left(\pi_{i}^{k}\right)_{i \in S}, k=0, \ldots, T-1$, are just the family of periodically invariant distributions of $\xi$.

Summing the last equation over $i_{2}, \ldots, i_{T}$ yields $\pi_{i_{0}}^{k} p_{i_{0} i_{1}}(k, k+1)=\pi_{i_{1}}^{k-1} p_{i_{1} i_{0}}(k-1, k)$. Summing (5.2) over $i_{0}, i_{3}, \ldots, i_{T}$ yields $\pi_{i_{1}}^{k+1} p_{i_{1} i_{2}}(k+1, k+2)=\pi_{i_{2}}^{k-2} p_{i_{2} i_{1}}(k-2, k-1)$. So $\pi_{i_{1}}^{k} p_{i_{1} i_{2}}(k, k+1)=\pi_{i_{2}}^{k-3} p_{i_{2} i_{1}}(k-3, k-2)$, because of the arbitrariness of $k$. Therefore,

$$
\pi_{i}^{k-1} p_{i j}(k-1, k)=\pi_{i}^{k-3} p_{i j}(k-3, k-2) \quad \text { for all } i, j \in S .
$$

This implies that $\pi_{i_{1}}^{k-1}=\pi_{i_{1}}^{k-3}$. Hence, $P_{k-1}=P_{k-3}$ for all $k$.

Remark 5.2. In fact, when $\left\{\xi_{n}: n=0,1, \ldots\right\}$ is completely periodically reversible, it must have period two.

\subsection{Discrete-time Markov chains}

Here we state some analogous results, whose proofs are quite simple.

Definition 5.1. If an inhomogeneous Markov chain $\xi=\left\{\xi_{n}: n=0,1, \ldots\right\}$ is such that $\left(\xi_{k}, \xi_{k+1}\right)$ and $\left(\xi_{k+1}, \xi_{k}\right)$ have the same distribution, then we say that $\xi$ is instantaneously reversible at time $k$. If $\xi$ is instantaneously reversible at all times, then we say that $\xi$ is instantaneously reversible.

Definition 5.2. We define the instantaneous entropy production rate, $e_{\mathrm{p}}^{k}$, of $\xi$ at time $k$ as

$$
e_{\mathrm{p}}^{k}=H\left(\mathrm{P}_{[k, k+1]}, \mathrm{P}_{[k, k+1]}^{-}\right),
$$

where $\mathrm{P}_{[k, k+1]}$ is the distribution of $\left(\xi_{k}, \xi_{k+1}\right), \mathrm{P}_{[k, k+1]}^{-}$is the distribution of $\left(\xi_{k+1}, \xi_{k}\right)$, and $H\left(\mathrm{P}_{[k, k+1]}, \mathrm{P}_{[k, k+1]}^{-}\right)$is the relative entropy of $\mathrm{P}_{[k, k+1]}$ with respect to $\mathrm{P}_{[k, k+1]}^{-}$.

Proposition 5.2. The instantaneous entropy production rate $e_{\mathrm{p}}^{k}$ can be expressed as

$$
\frac{1}{2} \sum_{i, j \in S}\left(\pi_{i}^{k} p_{i j}^{k}-\pi_{j}^{k} p_{j i}^{k}\right) \log \frac{\pi_{i}^{k} p_{i j}^{k}}{\pi_{j}^{k} p_{j i}^{k}} .
$$

The main result in this case is as follows.

Theorem 5.2. The following statements are equivalent, in close accord with physical theory.

1. $\xi$ is instantaneously reversible at time $k$.

2. It is in detailed balance: $\pi_{i}^{k} p_{i j}^{k}=\pi_{j}^{k} p_{j i}^{k}$ for all $i, j \in S$.

3. Its instantaneous entropy production rate at time $k$ vanishes. 
Corollary 5.1. The inhomogeneous Markov chain $\xi$ is instantaneously reversible if and only if each of the transition probability matrices $P(n)$ is reversible when it is regarded as the transition probability matrix of a homogeneous Markov chain and their reversible distributions are the same.

Remark 5.3. The concepts of instantaneous reversibility and periodic reversibility are not equivalent, and neither is stronger than the other.

\section{Acknowledgements}

The author would like to thank the anonymous referee for helpful suggestions and the editor for revising the English presentation. The support of the 973 Funds of China for Nonlinear Science and NSFC 10271008 are acknowledged. This work was completed by D. Q. Jiang at the Institute of Applied Mathematics, University of Bonn. Its hospitality and the support of the Alexander von Humboldt-Stiftung are gratefully acknowledged.

\section{References}

[1] Arizmendi, C. M. And Family, F. (1999). Algorithmic complexity and efficiency of a ratchet. Physica A 269, 285-292.

[2] Astumian, R. D. (1997). Thermodynamics and kinetics of a Brownian motor. Science 276, 917-922.

[3] Astumian, R. D. And Bier, M. (1994). Fluctuation driven ratchets: molecular motors. Phys. Rev. Lett. 72, 1766-1769.

[4] Berdichevsky, V. And Gitterman, M. (1998). Stochastic resonance and ratchets - new manifestations. Physica A 249, 88-95.

[5] Bier, M. (1997). Brownian ratchets in physics and biology. Contemp. Phys. 38, 371-379.

[6] Dollard, J. D. And Friedman, C. N. (1979). Product Integration with Applications to Differential Equations (Encyclopaedia Math. Appl. 10). Addison-Wesley, Reading, MA.

[7] Elston, T. C. And Doering, C. R. (1996). Numerical and analytical studies of nonequilibrium fluctuationinduced transport processes. J. Statist. Phys. 83, 359-383.

[8] Gammaitoni, L., Hänggi, P., Jung, P. And Marchesoni, F. (1998). Stochastic resonance. Rev. Mod. Phys. 70, $223-287$.

[9] Ge, H., JiAng, D. Q. And Qian, M. (2006). A simple discrete model of Brownian motors: time-periodic Markov chains. J. Statist. Phys. 123, 831-859.

[10] Hasegawa, H. (1976). On the construction of a time-reversed Markoff process. Progress Theoret. Phys. 55, 90-105.

[11] Hasegawa, H. (1976). Variational principle for non-equilibrium states and the Onsager-Machlup formula. Progress Theoret. Phys. 56, 44-60.

[12] Hasegawa, H. (1977). Thermodynamic properties of non-equilibrium states subject to Fokker-Planck equations. Progress Theoret. Phys. 57, 1523-1537.

[13] Hasegawa, H. (1977). Variational approach in studies with Fokker-Planck equations. Progress Theoret. Phys. 58, 128-146.

[14] Hu, D. H. (1983). Markov Process with Countable State Space. Wuhan University Press (in Chinese).

[15] Jiang, D. Q., Qian, M. AND QIan, M. P. (2004). Mathematical Theory of Nonequilibrium Steady States. On the Frontier of Probability and Dynamical Systems (Lecture Notes Math. 1833). Springer, Berlin.

[16] Jülicher, F., Ajdari, A. AND Prost, J. (1997). Modeling molecular motors. Rev. Mod. Phys. 69, 1269-1281.

[17] Lin, X., Zhang, H. J. And Hou, Z. T. (2000). The property of transition matrix of nonhomogeneous Markov chains. J. Changsha Railway Univ. 18, 86-90 (in Chinese).

[18] Magnasco, M. O. (1993). Forced thermal ratchets. Phys. Rev. Lett. 71, 1477-1481.

[19] Magnasco, M. O. (1994). Molecular combustion motors. Phys. Rev. Lett. 72, 2656-2659.

[20] Nicolis, G. and Prigogine, I. (1997). Self-Organization in Nonequilibrium Systems. From Dissipative Structures to Order through Fluctuations. John Wiley, New York.

[21] QIAN, M. P. AND QIAN, M. (1982). Circulation for recurrent Markov chains. Z. Wahrscheinlichkeitsth. 59, 203-210.

[22] QIan, M. P. And QIan, M. (1985). The entropy production and reversibility of Markov processes. Sci. Bull. 30, 165-167.

[23] QIAN, M. P., QIAN, M. AND Gong, G. L. (1991). The reversibility and the entropy production of Markov processes. Contemp. Math. 118, 255-261. 
[24] Qian, M. P., QIAN, C. AND QIAN, M. (1984). Circulations of Markov chains with continuous time and the probability interpretation of some determinants. Sci. Sinica A 27, 470-481.

[25] Reimann, P. (2002). Brownian motors: noisy transport far from equilibrium. Phys. Rep. 361, 57-265.

[26] Schnakenberg, J. (1976). Network theory of microscopic and macroscopic behavior of master equation systems. Rev. Mod. Phys. 48, 571-585.

[27] Walters, P. (1982). An Introduction to Ergodic Theory (Grad. Texts Math. 79). Springer, New York.

[28] Wang, H., Peskin, C. S. and Elston, T. C. (2003). A robust numerical algorithm for studying biomolecular transport processes. J. Theoret. Biol. 221, 491-511.

[29] Xing, J., WANG, H. Y. AND Oster, G. (2005). From continuum Fokker-Planck models to discrete kinetic models. Biophys. J. 89, 1551-1563.

[30] Yin, G. G. And Zhang, Q. (1998). Continuous-Time Markov Chains and Applications. A Singular Perturbation Approach (Appl. Math. 37). Springer, Berlin.

[31] Zhang, F. X. (2005). Exclusion processes on groups: entropy production density and reversibility. Physica A 348, 131-139. 